\title{
A NOTE ON POINTWISE CONVERGENCE FOR THE SCHRÖDINGER EQUATION
}

\author{
RENATO LUCÀ AND KEITH M. ROGERS
}

\begin{abstract}
We consider Carleson's problem regarding pointwise convergence for the Schrödinger equation. Bourgain recently proved that there is initial data, in $H^{s}\left(\mathbb{R}^{n}\right)$ with $s<\frac{n}{2(n+1)}$, for which the solution diverges on a set of nonzero Lebesgue measure. We provide a different example enabling the generalisation to fractional Hausdorff measure.
\end{abstract}

\section{INTRODUCTION}

Consider the Schrödinger equation, $i \partial_{t} u+\Delta u=0$, in $\mathbb{R}^{n+1}$, with initial data $u(\cdot, 0)=u_{0}$ in the Bessel potential/Sobolev space defined by

$$
H^{s}\left(\mathbb{R}^{n}\right)=(1-\Delta)^{-s / 2} L^{2}\left(\mathbb{R}^{n}\right):=\left\{G_{s} * g: g \in L^{2}\left(\mathbb{R}^{n}\right)\right\} .
$$

The Bessel kernel $G_{s}$ is defined as usual via its Fourier transform; $\widehat{G}_{s}=\left(1+|\cdot|^{2}\right)^{-s / 2}$. In [5], Carleson proposed the problem of identifying the exponents $s>0$ for which

$$
\lim _{t \rightarrow 0} u(x, t)=u_{0}(x), \quad \text { a.e. } \quad x \in \mathbb{R}^{n}, \quad \forall u_{0} \in H^{s}\left(\mathbb{R}^{n}\right),
$$

with respect to Lebesgue measure, and proved that (1.1) holds as long as $s \geq 1 / 4$ and $n=1$. Dahlberg and Kenig then showed that this condition is necessary, providing a complete solution in the one-dimensional case [6].

In higher dimensions, (1.1) holds as long as $s>\frac{2 n-1}{4 n}$; see [12, 3]. It was thought that $s \geq 1 / 4$ might also be sufficient in higher dimensions (see for example [11] or [19]), however Bourgain recently proved that $s \geq \frac{n}{2(n+1)}$ is necessary [4]. Since then, Du, Guth and Li 8 improved the sufficient condition in two dimensions to the almost $\operatorname{sharp} s>1 / 3$.

Here we give a new proof of the necessary condition using a different example (fewer frequencies travelling in a skew direction; see (3.2)). We replace number theoretic arguments, via comparison with Guass sums, with ergodic arguments that exploit the occasional complete absence of cancelation as in [13. This permits us to generalise to fractional Hausdorff measure. When $n=2$, the proof becomes much simpler as the ergodic arguments are trivial in that case.

Theorem 1.1. Let $(3 n+1) / 4 \leq \alpha \leq n$. Then, for any

$$
s<\frac{n}{2(n+1)}+\frac{n-1}{2(n+1)}(n-\alpha)
$$

there exists $u_{0} \in H^{s}\left(\mathbb{R}^{n}\right)$ such that

$$
\limsup _{t \rightarrow 0}|u(x, t)|=\infty
$$

Supported by the ERC grants 277778 and 676675, the MINECO grants SEV-2015-0554 and MTM2013-41780-P (Spain), and the NSF grant DMS-1440140 (MSRI, Berkeley, Spring 2017). 
for all $x$ in a set of positive $\alpha$-Hausdorff measure.

The study of this refined version of Carleson's problem was initiated by Sjögren and Sjölin [17. Theorem 1.1] improves [13, Theorem 2], although the result there holds for the full range $n / 2 \leq \alpha \leq n$ (with $\alpha<n / 2$ the question was previously resolved in [1]). It has been conjectured that $s \geq \frac{n}{2(n+1)}$ should also be sufficient in the $\alpha=n$ case; see [7. If that were true, then (1.2) would represent the interpolating condition between two sharp results, and so it would be interesting to see if Theorem 1.1 could be extended to the range $n / 2 \leq \alpha \leq n$, or whether there is a discontinuity in behaviour as in the one-dimensional case.

Indeed, defining

$$
\alpha_{n}(s):=\sup _{u_{0} \in H^{s}\left(\mathbb{R}^{n}\right)} \operatorname{dim}\left\{x \in \mathbb{R}^{n}: \limsup _{t \rightarrow 0}|u(x, t)|=\infty\right\},
$$

where dim denotes the Hausdorff dimension, the combination of Theorem 1.1 with previous results yields

$$
\alpha_{n}(s) \geq\left\{\begin{array}{clrl}
n & \text { when } & s<\frac{n}{2(n+1)} \\
n+\frac{n}{n-1}-\frac{2(n+1) s}{n-1} & \text { when } \frac{n}{2(n+1)} \leq s<\frac{n+1}{8} \\
n+1-\frac{2(n+2) s}{n} & \text { when } \frac{n+1}{8} \leq s<\frac{n}{4} \\
n-2 s & \text { when } \frac{n}{4} \leq s \leq \frac{n}{2} .
\end{array}\right.
$$

The function on the right-hand side is continuous apart from a jump of $\frac{1}{2 n}$ over the regularity $s=\frac{n+1}{8}$. The bound is best possible in one dimension, in which case the central intervals are empty and the dimension jumps by a half over $s=1 / 4$. This is a consequence of the Dahlberg-Kenig example combined with [1], where it was proven that $\alpha_{n}(s) \leq n-2 s$ in the range $n / 4 \leq s \leq n / 2$. For the best known upper bounds with lower regularity, see [14, Theorem 1.2].

In the following section we present the quantitive ergodic lemma that will be used in the third section to provide a new proof that $s \geq \frac{n}{2(n+1)}$ is necessary in the Lebesgue measure case. For this we will employ the Nikišin-Stein maximal principle. However, in the fourth section, we will explicitly construct data for which the divergence occurs, see (4.2), enabling the proof of Theorem 1.1 .

\section{A QUANTITIVE ERGODIC LEMMA}

It is well-known that linear flow on the torus, in most directions, eventually passes arbitrarily close to every point. This remains true when only considering equidistant points on the trajectory.

Lemma 2.1. Let $d \geq 2,0<\varepsilon, \delta<1$ and $\kappa>\frac{1}{d+1}$. Then, if $\delta<\kappa$ and $R>1$ is sufficiently large, there is $\theta \in \mathbb{S}^{d-1}$ for which, given any $y \in \mathbb{T}^{d}$ and $a \in \mathbb{R}$, there is $a t_{y} \in R^{\delta} \mathbb{Z} \cap(a, a+R)$ such that

$$
\left|y-t_{y} \theta\right| \leq \varepsilon R^{(\kappa-1) / d} .
$$

Moreover, this remains true with $d=1$, for some $\theta \in(0,1)$.

Proof. When $d=1$, by taking $\theta$ close to $R^{-1}$, we obtain approximately $R^{1-\delta}$ points $t_{y} \theta$ equally spaced at intervals of length $R^{\delta-1}$ on the circle. For each $y \in \mathbb{T}$, one of these points $t_{y} \theta$ must lie closer than a distance of $\varepsilon R^{\kappa-1}$ if $R$ is sufficiently large so that $R^{\delta-\kappa}<\varepsilon$. 
When $d \geq 2$ and $a=0$, this was proved in [13, Lemma 2]. The adjustment to the general case $a \in \mathbb{R}$ amounts to little more than starting the flow at different points on the translation invariant torus. One can also easily check that the proof in [13] is essentially unchanged. One need only translate their function $\eta_{R}$ by $a$, and the modulus of the Fourier transform of this is unchanged, so the remainder of the argument is exactly the same.

The following corollary is optimal, in the sense that the statement fails for larger $\sigma$. To see this, we can place balls of radius $\varepsilon R^{-\frac{\gamma}{d}}$ centred at the points of the sets below and assume that the balls are disjoint. Then the volume of such a set would be of the order $\varepsilon^{d} R^{d+1 / 2-\gamma-(d+2) \sigma}$, a quantity that is arbitrarily small for larger $\sigma$. Neither is it possible to extend the range of $\gamma$, as then the set of times $t$ could be empty. To avoid this we must have $\sigma<1 / 4$ which is ensured by the restriction $\gamma \geq 3 d / 4$.

Corollary 2.2. Let $d \geq 2, \frac{3 d}{4} \leq \gamma \leq d$ and $0<\sigma<\frac{1+2(d-\gamma)}{2(d+2)}$. Then, for any $\varepsilon>0$ and sufficiently large $R>1$, there exists $\theta \in \mathbb{S}^{d-1}$ such that

$$
\bigcup_{t \in R^{2 \sigma-1} \mathbb{Z} \cap\left(a, a+R^{-1 / 2}\right)}\left\{x \in R^{\sigma-1} \mathbb{Z}^{d}:|x| \leq 2\right\}+t \theta
$$

is $\varepsilon R^{-\frac{\gamma}{d}}$-dense in $B(0,1 / 2)$, for all $a \in(0,1)$. Moreover, this remains true with $d=1$, for some $\theta \in(0,1)$.

Proof. We first rescale by $R^{1-\sigma}$, and then replace $R^{1 / 2-\sigma}$ by $R$. In this way the statement is equivalent to proving that, for any $y \in B\left(0, R^{\frac{1-\sigma}{1 / 2-\sigma}} / 2\right)$ there exists

$$
x_{y} \in \mathbb{Z}^{d} \cap B\left(0,2 R^{\frac{1-\sigma}{1 / 2-\sigma}}\right) \quad \text { and } \quad t_{y} \in R^{\frac{\sigma}{1 / 2-\sigma}} \mathbb{Z} \cap\left(R^{\frac{1-\sigma}{1 / 2-\sigma}} a, R^{\frac{1-\sigma}{1 / 2-\sigma}} a+R\right)
$$

such that

$$
\left|y-\left(x_{y}+t_{y} \theta\right)\right|<\varepsilon R^{\frac{1-\sigma}{1 / 2-\sigma}-\frac{\gamma}{d(1 / 2-\sigma)}},
$$

for a fixed $\theta \in \mathbb{S}^{d-1}$, independent of $y$ and $a$. By taking the quotient $\mathbb{R}^{n} / \mathbb{Z}^{d}=\mathbb{T}^{d}$, this would follow if, for any $[y] \in \mathbb{T}^{d}$, we have

$$
\left|[y]-\left[t_{y} \theta\right]\right|<\varepsilon R^{\frac{1-\sigma}{1 / 2-\sigma}-\frac{\gamma}{d(1 / 2-\sigma)}} .
$$

Now this is a consequence of Lemma 2.1, by taking $\delta=\sigma /(1 / 2-\sigma)$ and $\kappa$ so that

$$
\frac{\kappa-1}{d}=\frac{1-\sigma}{1 / 2-\sigma}-\frac{\gamma}{d(1 / 2-\sigma)} .
$$

The conditions $0<\delta<1$ and $\delta<\kappa$ are then ensured by the restrictions on $\gamma$ and $\sigma$ in the statement.

\section{Proof of the Lebesgue measure neCessary CONDition}

When the initial data $u_{0}$ is a Schwartz function, the solution $u$ to the Schrödinger equation can be written as

$$
u(x, t)=e^{i t \Delta} u_{0}(x):=\frac{1}{(2 \pi)^{n / 2}} \int_{\mathbb{R}^{n}} \widehat{u}_{0}(\xi) e^{i x \cdot \xi-i t|\xi|^{2}} d \xi .
$$

By the Nikišin-Stein maximal principle [16, 18, it suffices to prove the following theorem. 
Theorem 3.1. Suppose that there is a constant $C_{s}$ such that

$$
\left\|\sup _{0<t<1}\left|e^{i t \Delta} f\right|\right\|_{L^{2}(B(0,1))} \leq C_{s}\|f\|_{H^{s}\left(\mathbb{R}^{n}\right)},
$$

whenever $f$ is a Schwartz function. Then $s \geq \frac{n}{2(n+1)}$.

Proof. Writing $t /(2 \pi R)$ in place of $t$, the maximal estimate implies that 1

$$
\left\|\sup _{0<t<1}\left|e^{i \frac{t}{2 \pi R} \Delta} f\right|\right\|_{L^{2}(B(0,1))} \lesssim R^{s}\|f\|_{2},
$$

whenever supp $\widehat{f} \subset B(0,2 R)$ and $R>4$. From now on we let $B(0, \rho)$ denote the $(n-1)$-dimensional ball of radius $\rho>0$, a fixed, sufficiently small constant. Writing $x=\left(x_{1}, \bar{x}\right)$ and letting $0<\sigma<\frac{1}{2(n+1)}$, we consider frequencies in the set

$$
\Omega:=\left\{\bar{\xi} \in 2 \pi R^{1-\sigma} \mathbb{Z}^{n-1}:|\bar{\xi}| \leq R\right\}+B(0, \rho),
$$

and Schwartz functions defined by $\widehat{\phi}=\chi_{(-\rho, \rho)}$ and $\widehat{g}=\chi_{\Omega}$. Then the initial data is defined by

$$
f(x)=e^{i \pi R(1, \theta) \cdot x} \phi\left(R^{1 / 2} x_{1}\right) g(\bar{x}),
$$

where $\theta \in(0,1)$ when $n=2$ and $\theta \in \mathbb{S}^{n-2}$ in higher dimensions.

Note that the solution factorises

$$
e^{i \frac{t}{2 \pi R} \Delta} f(x)=e^{i \frac{t}{2 \pi R} \Delta} f_{d k}\left(x_{1}\right) e^{i \frac{t}{2 \pi R} \Delta} f_{\theta}(\bar{x})
$$

where $f_{d k}$ and $f_{\theta}$ are defined by

$$
f_{d k}\left(x_{1}\right)=e^{i \pi R x_{1}} \phi\left(R^{1 / 2} x_{1}\right) \quad \text { and } \quad f_{\theta}(\bar{x})=e^{i \pi R \theta \cdot \bar{x}} g(\bar{x}) .
$$

By a change of variables, we have

$$
\begin{aligned}
\left|e^{i \frac{t}{2 \pi R} \Delta} f_{d k}\left(x_{1}\right)\right| & =\frac{R^{-1 / 2}}{(2 \pi)^{1 / 2}}\left|\int \widehat{\phi}\left(R^{-1 / 2}\left(\xi_{1}-\pi R\right)\right) e^{i x_{1} \xi_{1}-i \frac{t}{2 \pi R} \xi_{1}^{2}} d \xi_{1}\right| \\
& =\frac{1}{(2 \pi)^{1 / 2}}\left|\int_{-\rho}^{\rho} e^{i R^{1 / 2}\left(x_{1}-t\right) y-i \frac{t}{2 \pi} y^{2}} d y\right| \simeq 1
\end{aligned}
$$

whenever $|t| \leq 1$ and $\left|x_{1}-t\right| \leq R^{-1 / 2}$. Indeed, these restrictions ensure that the phase is close to zero, so that no cancelation occurs in the integral. By Plancherel's identity and Fubini's theorem,

$$
\|f\|_{2}=\left\|f_{d k}\right\|_{2}\left\|f_{\theta}\right\|_{2} \simeq R^{-1 / 4}|\Omega|^{1 / 2}
$$

so that plugging the data into the maximal estimate (3.1) and using (3.3) and (3.4), we obtain

$$
\left(\int_{B(0,1)} \int_{0}^{1 / 2} \sup _{t \in\left(x_{1}, x_{1}+R^{-1 / 2}\right)}\left|e^{i \frac{t}{2 \pi R} \Delta} f_{\theta}(\bar{x})\right|^{2} d x_{1} d \bar{x}\right)^{1 / 2} \lesssim R^{s} R^{-1 / 4}|\Omega|^{1 / 2} .
$$

In order to understand the behaviour of $e^{i \frac{t}{2 \pi R} \Delta} f_{\theta}$ we first consider the unmodulated version $e^{i \frac{t}{2 \pi R} \Delta} g$. Barceló, Bennett, Carbery, Ruiz and Vilela [2] showed that

$$
\left|e^{i \frac{t}{2 \pi R} \Delta} g(\bar{x})\right| \gtrsim|\Omega|, \quad \text { for all } \quad(\bar{x}, t) \in X_{0} \times R^{2 \sigma-1} \mathbb{Z} \cap(0,1),
$$

\footnotetext{
${ }^{1}$ We write $a \lesssim b(a \gtrsim b)$ whenever $a$ and $b$ are nonnegative quantities that satisfy $a \leq C b$ $(a \geq C b)$ for a constant $C>0$. We write $a \simeq b$ when $a \lesssim b$ and $b \lesssim a$.
} 
where, with $\varepsilon$ sufficiently small, $X_{0}$ is defined by

$$
X_{0}=\left\{\bar{x} \in R^{\sigma-1} \mathbb{Z}^{n-1}:|\bar{x}| \leq 2\right\}+B\left(0, \varepsilon R^{-1}\right) .
$$

This time the phase in the integrand never strays too far from zero modulo $2 \pi i$, and so again there is no cancelation in the integral. Now

$$
(\bar{x}, t) \in X_{t \theta} \times R^{2 \sigma-1} \mathbb{Z} \cap(0,1) \quad \Rightarrow \quad(\bar{x}-t \theta, t) \in X_{0} \times R^{2 \sigma-1} \mathbb{Z} \cap(0,1),
$$

where $X_{t \theta}:=X_{0}+t \theta$ and $\left|e^{i \frac{t}{2 \pi R} \Delta} f_{\theta}(\bar{x})\right|=\left|e^{i \frac{t}{2 \pi R} \Delta} g(\bar{x}-t \theta)\right|$. Combining this fact with (3.6) yields

$$
\sup _{t \in\left(x_{1}, x_{1}+R^{-1 / 2}\right)}\left|e^{i \frac{t}{2 \pi R} \Delta} f_{\theta}(\bar{x})\right| \gtrsim|\Omega|, \quad \text { for all } \quad \bar{x} \in \Gamma_{x_{1}}:=\underbrace{}_{t \in R^{2 \sigma-1}} \bigcup_{\mathbb{Z} \cap\left(x_{1}, x_{1}+R^{-1 / 2}\right)} X_{t \theta},
$$

and this holds uniformly for all $x_{1} \in(0,1 / 2)$.

Now the sets $\Gamma_{x_{1}}$ can be considered to be $\varepsilon R^{-1}$-neighbourhoods of the sets of Corollary 2.2. So, taking $\gamma=d=n-1$, there is a $\theta \in \mathbb{S}^{n-2}$ so that $B(0,1 / 2) \subset \Gamma_{x_{1}}$ for all $x_{1} \in(0,1 / 2)$. Substituting into (3.5), this yields

$$
|\Omega|^{1 / 2} \lesssim R^{s} R^{-1 / 4}
$$

As $|\Omega| \simeq R^{(n-1) \sigma}$, we can let $\sigma$ tend to $\frac{1}{2(n+1)}$ and then $R$ tend to infinity, so that

$$
s \geq 1 / 4+\frac{n-1}{4(n+1)}=\frac{n}{2(n+1)},
$$

which completes the proof.

\section{Proof of Theorem 1.1}

The solution is typically represented as $u(\cdot, t):=\lim _{N \rightarrow \infty} S_{N}(t) u_{0}$, where

$$
S_{N}(t) u_{0}(x):=\frac{1}{(2 \pi)^{n / 2}} \int_{\mathbb{R}^{n}} \Psi\left(N^{-1} \xi\right) \widehat{u}_{0}(\xi) e^{i x \cdot \xi-i t|\xi|^{2}} d \xi,
$$

and $\Psi$ is a fixed function, equal to one near the origin, that decays in such a way that the integral is well-defined. For convenience we take $\Psi(\xi)=\prod_{j=1}^{n} \psi\left(\xi_{j}\right)$, where $\psi$ is differentiable, supported in the interval $[-2,2]$ and equal to one on $[-1,1]$. The limit is usually taken with respect to the $L^{2}$-norm, but here we will take all limits pointwise, at each point that they exist. Supposing that $\alpha>n-2 s$, as we may, the limits exist at almost every $x$ with respect to $\alpha$-Hausdorff measure (see for example [15. Corollary 17.6]) and they coincide with the usual $L^{2}$-limit almost everywhere with respect to Lebesgue measure.

We take $0<\sigma<\frac{1+2(n-\alpha)}{2(n+1)}$ and $\lambda:=2^{\frac{M}{1-\sigma}}$, with $M \in \mathbb{Z}$ to be chosen sufficiently large later. As $\alpha \geq(3 n+1) / 4$ we have that $\sigma<1 / 4$. Writing $\xi=\left(\xi_{1}, \bar{\xi}\right)$, we consider the sets of frequencies

$$
\Omega^{j}=\left\{\bar{\xi} \in 2 \pi \lambda^{j(1-\sigma)} \mathbb{Z}^{n-1}: \lambda^{j} \leq\left|\xi_{m}\right|<\lambda^{j+1}, m=2, \ldots, n\right\}+Q\left(0, \frac{\varepsilon_{1}}{\sqrt{n-1}}\right),
$$

where $\varepsilon_{1}>0$ is a fixed sufficiently small constant and $j \in \mathbb{N}$. Here $Q(0, \ell)$ is the closed $(n-1)$-dimensional cube centred at the origin with side-length $\ell$, and we denote its interior by $\stackrel{\circ}{Q}(0, \ell)$. For a suitable choice of $\theta_{j} \in(0,1)$ when $n=2$ or 
$\theta_{j} \in \mathbb{S}^{n-2}$ in higher dimensions, the initial data $u_{0}$ that gives rise to a divergent solution is given by

$$
u_{0}(x):=\sum_{j \in \mathbb{N}} e^{i \pi \lambda^{j}\left(1, \theta_{j}\right) \cdot x} \phi\left(\lambda^{j / 2} x_{1}\right) g_{j}(\bar{x}) .
$$

Here $\widehat{\phi}=\chi_{\left(-\varepsilon_{1}, \varepsilon_{1}\right)}$ and $\widehat{g}_{j}:=\lambda^{j \delta}\left|\Omega^{j}\right|^{-1} \chi_{\Omega^{j}}$, with $0<\delta<\sigma / 4$. Noting that $\left|\Omega^{j}\right| \simeq \lambda^{j(n-1) \sigma}$, we have that $u_{0} \in H^{s}$ whenever

$$
s<\frac{(n-1) \sigma}{2}+\frac{1}{4}-\delta .
$$

Eventually we will let $\sigma$ tend to $\frac{1+2(n-\alpha)}{2(n+1)}$ and $\delta$ tend to zero, covering all the cases of the range (1.2).

First we consider $(n-1)$-dimensional data given by $f_{\theta_{j}}(\bar{x}):=e^{i \pi \lambda^{j} \theta_{j} \cdot \bar{x}} g_{j}(\bar{x})$ and the associated solutions on $X_{t \theta_{j}}^{j} \times T_{x_{1}}^{j}$ defined by

$$
\begin{gathered}
X_{t \theta_{j}}^{j}=\left\{\bar{x} \in \lambda^{j(\sigma-1)} \mathbb{Z}^{n-1}:|\bar{x}| \leq 2\right\}+\stackrel{\circ}{ }\left(t \theta_{j}, \varepsilon_{2} \lambda^{-j}\right), \\
T_{x_{1}}^{j}=\left\{t \in \lambda^{j(2 \sigma-1)} \mathbb{Z}: x_{1}<t<x_{1}+\lambda^{-j / 2}\right\} .
\end{gathered}
$$

Taking $\varepsilon_{1}, \varepsilon_{2}$ sufficiently small and $x_{1} \in(0,1 / 2)$, as in the previous section we have

$$
\left|S_{N}\left(\frac{t}{2 \pi \lambda^{j}}\right) f_{\theta_{j}}(\bar{x})\right| \gtrsim \lambda^{j \delta}, \quad \text { for all } \quad(\bar{x}, t) \in X_{t \theta_{j}}^{j} \times T_{x_{1}}^{j}
$$

whenever $N \geq 2 \pi \lambda^{2 j}$; see [13, eq. 18]. On the other hand, in [13, eq. 20] it was proven that for $k>2 j$, we have

$$
\left|S_{N}\left(\frac{t}{2 \pi \lambda^{j}}\right) f_{\theta_{k}}(\bar{x})\right| \lesssim \lambda^{-k \delta}, \quad \text { for all } \quad(\bar{x}, t) \in \mathbb{R}^{n-1} \times T_{x_{1}}^{j},
$$

whenever $N \geq 2 \pi \lambda^{2 j}$. It is something of a nuisance that this does not quite hold for all $k>j$. To circumvent this, we consider

$$
X_{\lambda^{k-j} t \theta_{k}}^{k, \delta}:=\lambda^{k(\sigma-1)} \mathbb{Z}^{n-1}+Q\left(\lambda^{k-j} t \theta_{k}, \varepsilon_{2} \lambda^{-k(1-2 \delta)}\right) .
$$

In [13, eq. 19] it was proven that, when $j<k \leq 2 j$ and $x_{1} \in(0,1 / 2)$,

$$
\left|S_{N}\left(\frac{t}{2 \pi \lambda^{j}}\right) f_{\theta_{k}}(\bar{x})\right| \lesssim \lambda^{-k \delta}, \quad \text { for all } \quad(\bar{x}, t) \in\left(\mathbb{R}^{n-1} \backslash X_{\lambda^{k-j} t \theta_{k}}^{k, \delta}\right) \times T_{x_{1}}^{j}
$$

whenever $N \geq 2 \pi \lambda^{2 j}$. Thus, considering

$$
\Gamma_{t \theta_{j}}^{j}:=X_{t \theta_{j}}^{j} \backslash \bigcup_{j<k \leq 2 j} X_{\lambda^{k-j} t \theta_{k}}^{k, \delta} \quad \text { and } \quad \Gamma_{x_{1}}^{j}:=\bigcup_{t \in T_{x_{1}}^{j}} \Gamma_{t \theta_{j}}^{j},
$$

an immediate consequence of (4.3), (4.5) and (4.4) is that if $x \in \Gamma^{j}$, defined by

$$
\Gamma^{j}=\left\{x \in \mathbb{R}^{n}: x_{1} \in(0,1 / 2), \quad \bar{x} \in \Gamma_{x_{1}}^{j}\right\},
$$

there exists a time $t_{j}(x) \in T_{x_{1}}^{j}$ such that

(i) $\left|S_{N}\left(\frac{t_{j}(x)}{2 \pi \lambda^{j}}\right) f_{\theta_{j}}(\bar{x})\right| \gtrsim \lambda^{j \delta} ; \quad$ (ii) $\left|S_{N}\left(\frac{t_{j}(x)}{2 \pi \lambda^{j}}\right) f_{\theta_{k}}(\bar{x})\right| \lesssim \lambda^{-k \delta} \quad$ for all $\quad k>j$.

Now divergence occurs on the set of $x$ that belong to infinitely many $\Gamma^{j}$; that is

$$
\Gamma:=\bigcap_{j \geq 1} \bigcup_{k \geq j} \Gamma^{k}
$$

To see this, we note that if $x \in \Gamma$ there exists an infinite subset $J(x) \subset \mathbb{N}$ with an associated sequence of times $t_{j}(x) \in T_{x_{1}}^{j}$, for all $j \in J(x)$, such that both (i) 
and (ii) are satisfied. The solution factorises as in (3.3), so that, recalling (3.4), we see that the properties (i) and (ii) remain true while considering the extension $f_{j}$, defined by

$$
f_{j}(x)=e^{i \pi \lambda^{j} x_{1}} \phi\left(\lambda^{j / 2} x_{1}\right) f_{\theta_{j}}(\bar{x}) .
$$

Now, since $u_{0}=\sum_{j \geq 1} f_{j}$, by the triangle inequality

$$
\left|S_{N}\left(\frac{t_{j}(x)}{2 \pi \lambda^{j}}\right) u_{0}(x)\right| \gtrsim\left|S_{N}\left(\frac{t_{j}(x)}{2 \pi \lambda^{j}}\right) f_{j}(x)\right|-\left|A_{1}\right|-\left|A_{2}\right|,
$$

where

$$
A_{1}:=\sum_{1 \leq k<j} S_{N}\left(\frac{t_{j}(x)}{2 \pi \lambda^{j}}\right) f_{k}(x) \quad \text { and } \quad A_{2}:=\sum_{k>j} S_{N}\left(\frac{t_{j}(x)}{2 \pi \lambda^{j}}\right) f_{k}(x) .
$$

We have already proved that, for $x \in \Gamma$,

$$
\left|S_{N}\left(\frac{t_{j}(x)}{2 \pi \lambda^{j}}\right) f_{j}(x)\right| \gtrsim \lambda^{j \delta} \quad \text { and } \quad\left|A_{2}\right| \leq \sum_{k>j} \lambda^{-k \delta} \lesssim 1 .
$$

On the other hand, by bounding the terms trivially and taking $\lambda$ sufficiently large, we can also arrange that

$$
\left|A_{1}\right| \leq \sum_{1 \leq k<j} \lambda^{k \delta} \leq \frac{1}{2}\left|S_{N}\left(\frac{t_{j}(x)}{2 \pi \lambda^{j}}\right) f_{j}(x)\right| .
$$

Thus, for any $x \in \Gamma$ where the solution is defined, we have

$$
\left|u\left(x, \frac{t_{j}(x)}{2 \pi \lambda^{j}}\right)\right|=\lim _{N \rightarrow \infty}\left|S_{N}\left(\frac{t_{j}(x)}{2 \pi \lambda^{j}}\right) u_{0}(x)\right| \gtrsim \lambda^{j \delta},
$$

so there is a sequence of times $\frac{t_{j}(x)}{2 \pi \lambda^{j}}$ for which

$$
\left|u\left(x, \frac{t_{j}(x)}{2 \pi \lambda^{j}}\right)\right| \rightarrow \infty \quad \text { as } \quad \frac{t_{j}(x)}{2 \pi \lambda^{j}} \rightarrow 0 .
$$

Now, recalling that $s<\frac{(n-1) \sigma}{2}+\frac{1}{4}-\delta$, the proof would be complete if we could prove that the $\alpha$-Hausdorff measure of $\Gamma$ were positive, taking $\delta$ and $\sigma$ sufficiently close to 0 and $\frac{1+2(n-\alpha)}{2(n+1)}$, respectively. Considering the slices $\Gamma_{x_{1}}$, defined via

$$
\Gamma=\left\{x \in \mathbb{R}^{n}: x_{1} \in(0,1 / 2), \quad \bar{x} \in \Gamma_{x_{1}}\right\},
$$

it would suffice to prove that the $(\alpha-1)$-Hausdorff measure of $\Gamma_{x_{1}}$ is positive for all $x_{1} \in(0,1 / 2)$; see for instance [10, Proposition 7.9]. For this we must choose the modulation directions $\theta_{j} \in \mathbb{S}^{n-2}$ appropriately, via the ergodic argument of the second section $\left(\theta_{j} \in(0,1)\right.$ if $\left.n=2\right)$. Note that $X_{t \theta_{j}}^{j}$ is a union of disjoint open cubes of side-length $\varepsilon_{2} \lambda^{-j}$, while $X_{\lambda^{k-j} t \theta_{k}}^{k, \delta}$ is a union of disjoint closed cubes of side-length $\varepsilon_{2} \lambda^{-(1-2 \delta) k}$. The distance between the cubes is approximately $\lambda^{(\sigma-1) j}$ in the case of the former and $\lambda^{(\sigma-1) k}$ in the case of the latter. Thus we see that $\Gamma_{t \theta_{j}}^{j}$ is a union of disjoint open sets $\mathcal{Q}\left(\bar{x}, \varepsilon_{2} \lambda^{-j}\right)$ that we call pseudo-cubes. 
Case $\alpha=n$. In this case, the $(n-1)$-dimensional Lebesgue measure $|\cdot|$ of the pseudo-cubes is comparable to actual cubes;

$$
\begin{aligned}
& \left|\mathcal{Q}\left(\bar{x}, \varepsilon_{2} \lambda^{-j}\right)\right| \geq\left|Q\left(\bar{x}, \varepsilon_{2} \lambda^{-j}\right)\right|-\left|Q\left(\bar{x}, \varepsilon_{2} \lambda^{-j}\right) \cap \bigcup_{j<k \leq 2 j} X_{\lambda^{k-j} t \theta_{k}}^{k, \delta}\right| \\
& \simeq \varepsilon_{2}^{n-1} \lambda^{-(n-1) j}-\varepsilon_{2}^{n-1} \lambda^{-(n-1) j} \sum_{k=j+1}^{2 j} \lambda^{-(n-1)(1-2 \delta) k} \lambda^{(n-1)(1-\sigma) k} \gtrsim \varepsilon_{2}^{n-1} \lambda^{-(n-1) j},
\end{aligned}
$$

where we have taken $\lambda$ sufficiently large (recalling $\delta<\sigma / 4$ ). Thus, using Corollary 2.2 with $d=n-1, \gamma=\alpha-1$ and $R=\lambda^{j}$, we can choose the $\theta_{j}$ so that $\left|\Gamma_{x_{1}}^{j}\right| \gtrsim 1$ for all $x_{1} \in(0,1 / 2)$, provided that $j$ is sufficiently large and $\sigma<\frac{n}{2(n+1)}$. From this we see that

$$
\lim _{j \rightarrow \infty}\left|\bigcup_{k \geq j} \Gamma_{x_{1}}^{k}\right| \gtrsim 1
$$

and, since this is a decreasing sequence of sets that are contained in a set with finite $(n-1)$-dimensional Lebesgue measure, we can conclude that

$$
\left|\Gamma_{x_{1}}\right|=\left|\bigcap_{j \geq 1} \bigcup_{k \geq j} \Gamma_{x_{1}}^{k}\right| \gtrsim 1
$$

for all $x_{1} \in(0,1 / 2)$. This completes the proof in the case $\alpha=n$.

Case $\alpha<n$. We will prove that the $\beta$-Hausdorff measure of $\Gamma_{x_{1}}$ is positive for any $\beta$ in the interval $\left(\frac{(n-1)(2 \alpha+1)}{2(n+1)}, \alpha-1\right)$. Note that the interval is not empty if we restrict to $\alpha>\frac{3 n+1}{4}$. This is enough to complete the proof, as we could have started with an $\alpha^{\prime}>\alpha \geq \frac{3 n+1}{4}$ that also satisfies

$$
s<\frac{n}{2(n+1)}+\frac{n-1}{2(n+1)}\left(n-\alpha^{\prime}\right),
$$

and performed all of the previous arguments for this $\alpha^{\prime}$.

Considering the Hausdorff content of a set $E \subset \mathbb{R}^{d}$ defined by

$$
\mathcal{H}_{\infty}^{\beta}(E):=\inf \left\{\sum_{i} \delta_{i}^{\beta}: E \subset \bigcup_{i} Q\left(x_{i}, \delta_{i}\right)\right\},
$$

by the triangle inequality as before, we have that

$$
\begin{aligned}
\mathcal{H}_{\infty}^{\beta}\left(\mathcal{Q}\left(\bar{x}, \varepsilon_{2} \lambda^{-j}\right)\right) & \geq \mathcal{H}_{\infty}^{\beta}\left(Q\left(\bar{x}, \varepsilon_{2} \lambda^{-j}\right)\right)-\mathcal{H}_{\infty}^{\beta}\left(Q\left(\bar{x}, \varepsilon_{2} \lambda^{-j}\right) \cap \bigcup_{j<k \leq 2 k} X_{\lambda^{k-j} t \theta_{k}}^{k, \delta}\right) \\
& \gtrsim \varepsilon_{2}^{\beta} \lambda^{-\beta j}-\varepsilon_{2}^{n-1} \lambda^{-(n-1) j} \sum_{k=j+1}^{2 j} \lambda^{-\beta(1-2 \delta) k} \lambda^{(n-1)(1-\sigma) k} \gtrsim \varepsilon_{2}^{\beta} \lambda^{-\beta j},
\end{aligned}
$$

using $(1-2 \delta) \beta-(n-1)(1-\sigma)>0$ and taking $\lambda$ sufficiently large. This holds, taking $\delta$ and $\sigma$ close enough to 0 and $\frac{1+2(n-\alpha)}{2(n+1)}$, respectively, since we have restricted to $\beta>\frac{(n-1)(2 \alpha+1)}{2(n+1)}$. Again we see that, in this range of $\beta$, the $\mathcal{H}_{\infty}^{\beta}$-content of the pseudo-cubes is comparable to that of the actual cubes.

We now use Corollary 2.2. with $d=n-1, \gamma=\alpha-1$ and $R=\lambda^{j}$, to choose $\theta_{j}$ such that, for all $x_{1} \in(0,1 / 2)$, the $\Gamma_{x_{1}}^{j}$ are unions of pseudo-cubes whose centres are $\varepsilon_{2} \lambda^{-j \frac{\alpha-1}{n-1}}$-dense in $B(0,1 / 2) \subset \mathbb{R}^{n-1}$, when $j$ is sufficiently large. Recalling that as the sidelengths are shorter, of length $\varepsilon_{2} \lambda^{-j}$, this is not enough to come 
close to covering the ball as before. However, discarding some pseudo-cubes, if necessary, we find that $\Gamma_{x_{1}}^{j}$ contains a set of pseudo-cubes whose centres are a quasi-lattice with separation $\lambda^{-j \frac{\alpha-1}{n-1}}$; see [13, Lemma 4]. That is to say, for any $\bar{y} \in$ $B(0,1 / 2) \cap \lambda^{-j \frac{\alpha-1}{n-1}} \mathbb{Z}^{n-1}$ there exists a unique centre $\bar{x}$ satisfying $|\bar{x}-\bar{y}| \leq \lambda^{-j \frac{\alpha-1}{n-1}}$.

Using a density theorem due to Falconer [9] (see also [10, Proposition 8.5] for a similar theorem), the positivity of the $\beta^{\prime}$-Hausdorff measure of $\Gamma_{x_{1}}$, for any $\beta^{\prime}<\beta$, is a consequence of the following density property

$$
\liminf _{j \rightarrow \infty} \mathcal{H}_{\infty}^{\beta}\left(\Gamma_{x_{1}}^{j} \cap Q(\bar{x}, \delta)\right) \geq c \delta^{\beta}, \quad \forall Q(\bar{x}, \delta) \subset B(0,1 / 2), \quad \forall \delta>0 .
$$

Thus it would be sufficient for us to show that (4.6) holds for $\beta \in\left(\frac{(n-1)(2 \alpha+1)}{2(n+1)}, \alpha-1\right)$. Essentially this means that the most efficient way to cover $\Gamma_{x_{1}}^{j} \cap Q(\bar{x}, \delta)$ is with a single cube of side $\delta$. The only real competitor is the cover that consists of the disjoint union of cubes of side-length $\varepsilon_{2} \lambda^{-j}$ placed on the top of the pseudo-cubes of the quasi-lattice. However, this cover is costed at

$$
\sum_{i} \delta_{i}^{\beta} \simeq\left(\frac{\delta}{\lambda^{-j \frac{\alpha-1}{n-1}}}\right)^{n-1}\left(\varepsilon_{2} \lambda^{-j}\right)^{\beta}=\varepsilon_{2}^{\beta} \delta^{n-1} \lambda^{j(\alpha-1-\beta)},
$$

which diverges as $j \rightarrow \infty$ (recalling that $\beta<\alpha-1$ ). The remaining coverings are ruled out in exactly the same way as in [13, Section 4]. The only requirement is that the $\mathcal{H}_{\infty}^{\beta}$-content of the pseudo-cubes is comparable to that of the actual cubes, which we have already observed, so the proof is complete.

\section{REFERENCES}

[1] J. A. Barceló, J. Bennett, A. Carbery and K. M. Rogers. On the dimension of diverence sets of dispersive equations, Math. Ann. 349 (2011), 599-622.

[2] J. A. Barceló, J. Bennett, A. Carbery, A. Ruiz and M. C. Vilela, Some special solutions of the Schrödinger equation, Indiana Univ. Math. J. 56 (2007), 1581-1593.

[3] J. Bourgain, On the Schrödinger maximal function in higher dimension, Tr. Mat. Inst. Steklova 280 (2013), 53-66.

[4] J. Bourgain, A note on the Schrödinger maximal function, J. Anal. Math. 130 (2016), 393396.

[5] L. Carleson, Some analytic problems related to statistical mechanics, in Euclidean harmonic analysis (Proc. Sem., Univ. Maryland, College Park, Md., 1979), 5-45, Lecture Notes in Math. 779, Springer, Berlin.

[6] B. E. J. Dahlberg and C. E. Kenig, A note on the almost everywhere behavior of solutions to the Schrödinger equation, in Harmonic analysis (Minneapolis, Minn., 1981), 205-209, Lecture Notes in Math. 908, Springer, Berlin.

[7] C. Demeter and S. Guo, Schrödinger maximal function estimates via the pseudoconformal transformation, (2016), arXiv:1608.07640 1 .

[8] X. Du, L. Guth and X. Li, A sharp Schrödinger maximal estimate in $\mathbb{R}^{2}$, (2016), arXiv:1612.08946

[9] K. Falconer, Classes of sets with large intersection, Mathematika 32 (1985), 191-205.

[10] K. Falconer, Fractal geometry: mathematical foundations and applications, Wiley, 2003.

[11] G. Gigante and F. Soria, On the boundedness in $H^{1 / 4}$ of the maximal square function associated with the Schrödinger equation, J. Lond. Math. Soc. 77 (2008), no. 1, 51-68.

[12] S. Lee, On pointwise convergence of the solutions to the Schrödinger equations in $\mathbb{R}^{2}$, Int. Math. Res. Not. (2006), 1-21.

[13] R. Lucà and K. M. Rogers, Coherence on fractals versus convergence for the Schrödinger equation, Comm. Math. Phys. 351 (2017), 341-359.

[14] R. Lucà and K. M. Rogers, Average decay for the Fourier transform of measures with applications, J. Eur. Math. Soc., to appear. 
[15] P. Mattila, Fourier analysis and Hausdorff dimension, Cambridge Studies in Advanced Mathematics 150, Cambridge Univ. Press, Cambridge, 2015.

[16] E. M. Nikišin, A resonance theorem and series in eigenfunctions of the Laplace operator, Izv. Akad. Nauk SSSR Ser. Mat. 36 (1972), 795-813.

[17] P. Sjögren and P. Sjölin, Convergence properties for the time-dependent Schrödinger equation, Ann. Acad. Sci. Fenn. 14 (1989), no. 1, 13-25.

[18] E. M. Stein, On limits of sequences of operators, Ann. of Math. 74 (1961), 140-170.

[19] T. Tao, A sharp bilinear restrictions estimate for paraboloids, Geom. Funct. Anal. 13 (2003), no. $6,1359-1384$.

Departement Matematik und Informatik, Universität Basel, 4051, Switzerland.

E-mail address: renato.luca@unibasel.ch

Instituto de Ciencias Matemáticas CSiC-UAM-UC3M-UCM, Madrid, 28049, Spain.

E-mail address: keith.rogers@icmat.es 